Gut, 1971, 12, 399-402

\title{
A paediatric tube and capsule for suction biopsy of the small intestinal mucosa designed for direct nasogastric intubation
}

\author{
R. E. SCHNEIDER AND R. CHANG \\ From the Institute of Nutrition of Central America and Panama (INCAP) and the Roosevelt Hospital, \\ University of San Carlos of Guatemala, Guatemala
}

SUMMARY The use of a modified paediatric tube and capsule for suction biopsy of the mucosa of the small intestine is discussed. The tube is small in diameter and can be introduced through the nose without local trauma and with minimal discomfort. The intubation technique was followed in 72 instances to obtain suction biopsies from the proximal mucosa of the small intestine in protein-calorie malnourished children, and the results obtained with this instrument are also presented.

Biopsies of the proximal mucosa of the small intestine are useful in the evaluation and diagnosis of several of the gastrointestinal disorders that affect children (Anderson, 1966). The Crosby paediatric capsule (Crosby and Kugler, 1957) and the paediatric multipurpose suction biopsy tube (Brandborg, Rubin, and Quinton, 1959) are the most popular instruments used to obtain small-intestinal biopsies. Both of them were initially used by the authors to study the changes present in the proximal small-intestinal mucosa of protein-calorie malnourished children. Due to difficulties experienced with the former instruments at the clinical centre of INCAP during intubation of patients over $1 \frac{1}{2}$ years of age, we decided to try out a modified tube and capsule developed in our laboratory. Although similar to the multipurpose suction biopsy tube, the small diameter permits its introduction through the nose without local trauma and with minimal discomfort. Thus, any damage to the tube walls by the children's teeth during transoral intubation can be avoided. At the same time, the emotional trauma experienced by the child during this procedure is lessened by proper sedation and the application of local anaesthesia to the nasal mucosa.

The purpose of this communication is to describe the general characteristics of the modified tube and capsule, the technique followed in 72 instances in which suction biopsies from the proximal smallintestinal mucosa of protein-calorie malnourished

Received for publication 2 December 1970. children were taken with this instrument, and the results obtained.

\section{Materials and Methods}

The children studied were between 13 and 69 months of age, and were protein-calorie malnourished. The majority of them were followed longitudinally, and suction biopsies of the duodenojejunal area were taken at different stages of their nutritional recovery while they were inpatients at the Metabolic Unit of the INCAP centre.

The modified tube consists of a $50 \mathrm{~cm}$ long no. 12 French Levin tube, into which a Bowden spring was tightly fitted. The lumen inside the spring was sufficient to allow the pullwire to move easily back and forth regardless of the different positions the tube might take during the intubation procedure. Due to the presence of small metal adaptors at the extremes of the tube, the handle and manometer from the original multipurpose suction biopsy tube ${ }^{1}$ could be adapted to its proximal end, while the new capsule, $13 \mathrm{~mm}$ long, with an O.D. of $2.7 \mathrm{~mm}$ and with two opposite eccentric holes, was fitted distally. The cylindrical knife was also reduced in size and diameter in order to ensure its proper and tightly adjusted movement inside the capsule (Fig. 1).

The tip of a no. 12 French Nelaton tube (approximately $6 \mathrm{~cm}$ in length) filled with mercury and sealed at both sides was attached to the distal ends of the

${ }^{1}$ Manufactured by W.E. Quinton Instrument Co, Seattle, Washington, USA. 


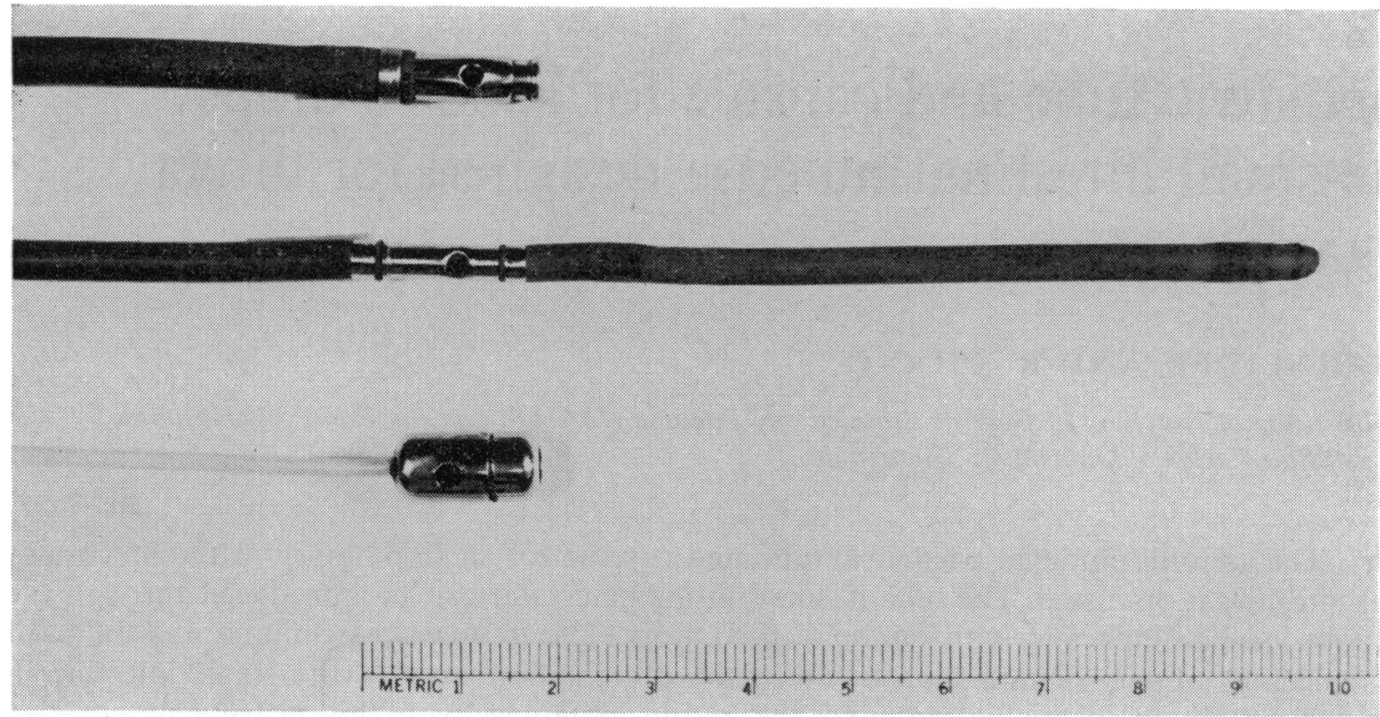

Fig. 1 Modified capsule compared with the paediatric multipurpose section biopsy (above) and the CrosbyKugler capsules (below). Note its smaller size and the tip of the Nelaton tube filled with mercury attached at the distal end of the capsule.

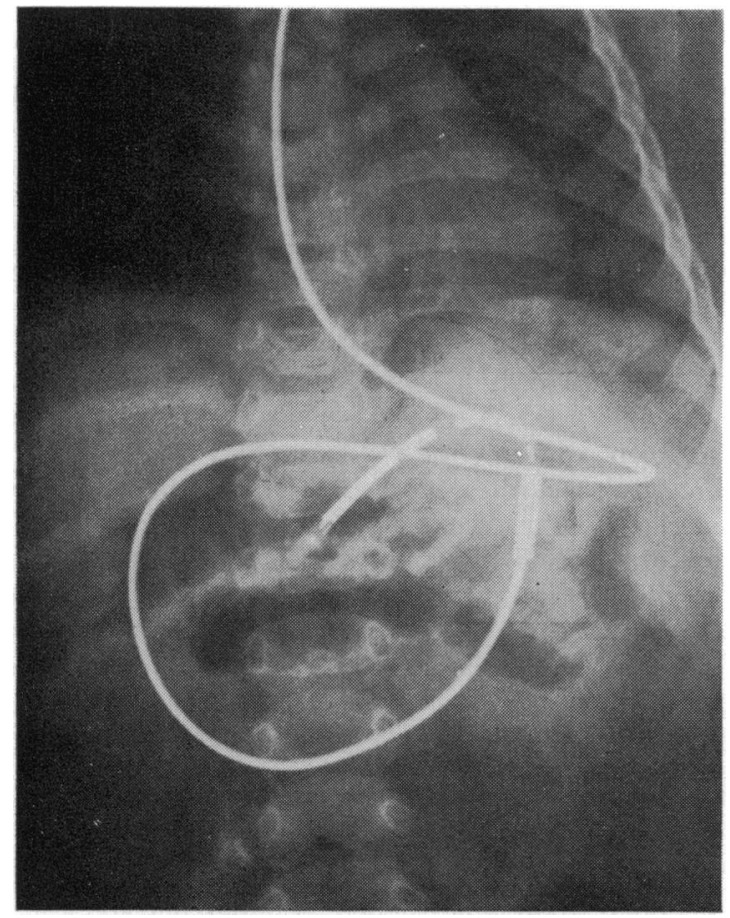

Fig. 2 Radiographic image showing the tube and rapsule in the ideal position to obtain biopsy specimens from the angle of Treitz. capsule. The extra weight of the mercury at the distal end of the tube was useful while manoeuvring the capsule during its passage through the stomach and duodenum. The Nelaton tip was flexible enough to decrease any injuries to the gastrointestinal mucosa during intubation.

After six hours of fasting the children were intubated under sedation using chloral hydrate (20 to $50 \mathrm{mg} / \mathrm{kg}$ of body weight) and metoclopramide ( 3 to $5 \mathrm{mg}$ ) orally in dosages which depended on their nutritional status. A small number of patients received a mixture of meperidine, chlorpromazine, and promethazine parenterally in the doses recommended by Israel, Hohn, Black, and Lambert (1967).

Local anaesthesia of the nasal mucosa helped to lessen the discomfort produced by the passage of the tube. A loose anterior tamponade was applied to each nasal fossa using thin strips of cotton moistened with $2 \%$ novesine or $4 \%$ xylocaine and $0.25 \%$ ephedrine for five to 10 minutes. Both nasal fossae were examined beforehand to eliminate local pathology. The progress of the tube was checked under fluoroscopy until the capsule arrived at the angle of Treitz where the biopsies were routinely taken (Fig. 2). The mechanical steps involved in obtaining the suction biopsies were similar to those required by the multipurpose suction biopsy tube (Brandborg, Rubin, and Quinton, 1959) except that the optimal negative pressure needed to produce the 
invagination of the intestinal mucosa into the capsule was $5 \mathrm{~mm}$ of mercury. Usually a $2.5 \mathrm{ml}$ syringe produced enough suction to obtain the negative pressure needed, provided that the tube's sealing system was intact.

When the tube was withdrawn, the capsule frequently got stuck while passing through the rhinopharynx (cavum) due to its length and rigidity. It was found that this problem could be solved by introducing an extraluminal guide into the nose and pressing it gently forward (Fig. 3). The guide also prevented erosions of the nasal mucosa due to the friction caused by the passage of the tube.
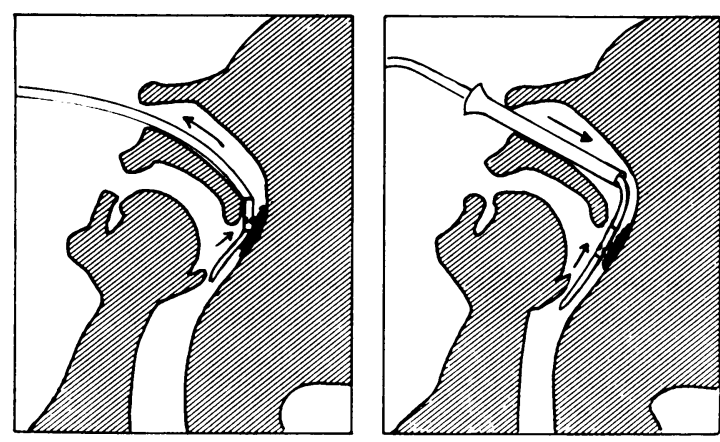

Fig. 3 The diagram shows how the extraluminal guide helps to prevent the capsule lodging while passing through the rhinopharynx during withdrawal of the tube. The dark area in the rhinopharynx simulates hypertrophied adenoid tissue.

\section{Results}

When the procedure was successful two specimens were obtained with the modified capsule in practically all instances. The average diameter of the biopsies when properly orientated and placed on a Marlex mesh was $2 \mathrm{~mm}$ ( 1.5 to $2.5 \mathrm{~mm}$ ). Histologically, the presence of a small rim of muscularis mucosae was observed in the majority of specimens studied.

With the modified tube and capsule, the failure rate was $21 \%$ ( 15 failures in 72 trials). This figure would be lower if the first 20 trials were disregarded, as the capsule and tube had to be modified to improve their performance. In any case, this is significantly lower than the $39 \%$ failure rate experienced with the original multipurpose suction biopsy tube. As the children's teeth were likely to damage the tube walls during transoral intubation it was sometimes impossible to exert the necessary negative pressure; this was the principal cause of failure with the original multipurpose suction biopsy tube.

No complications were experienced with the use of either tube. With the former, minor nasal bleeding was noted in a third of the patients, especially in those with hypertrophied adenoid tissue.

Certain aspects related to the use of sedation in protein-calorie malnourished children should be mentioned. In the first place, the application of a local anaesthetic to the nasal mucosa made it possible to administer smaller doses of the drugs used for sedation, as it decreases the discomfort produced by the tube's passage through the nose. No harmful effects were noticed with the application of either $2 \%$ novesine or $4 \%$ xylocaine. Secondly, similar levels of sedation were obtained with either the oral administration of chloral hydrate and metoclopramide or with a combination of meperidine, chlorpromazine, and promethazine administered parenterally. In the majority of the protein-calorie malnourished children, $20-25 \mathrm{mg}$ of chloral hydrate $/ \mathrm{kg}$ body weight and $2-3 \mathrm{mg}$ of clopropamide orally were enough to produce satisfactory levels of sedation 30-60 minutes after their administration. A 6-year-old girl was the only patient in whom a prolonged sedative effect was observed; she remained asleep for 15 hours, but without evidence of serious depression of the central nervous system, as the respiratory rate continued to be normal and she responded readily to painful stimuli. When fully recovered the children received $50 \mathrm{mg} / \mathrm{kg}$ body weight of chloral hydrate and $5 \mathrm{mg}$ of clopropamide. In many patients, particularly those in whom repeated biopsies were taken, chlorpromazine in dosages ranging from 3 to $8 \mathrm{mg}$ orally had to be added in order to obtain satisfactory sedation. Apparently these patients developed progressive resistance to the action of the drugs, for the number of instances when they were administered increased. This was sometimes observed when a second biopsy was taken a week after the first and while the child was still severely malnourished.

\section{Discussion}

During the initial trials several problems were encountered while using the Crosby paediatric capsule. In the first place, technical difficulties similar to those reported by other authors (Salem, Salt, and Truelove, 1965) were experienced with some frequency. Secondly, the children often had to be kept intubated from 10 to 16 hours before the capsule arrived at the site chosen for obtaining the biopsy. Finally, as transoral intubation was not possible, retrograde intubation was used and this 
was rather cumbersome and emotionally traumatic for the child. Retrograde intubation consisted in the passage, under sedation, of a no. 12 Nelaton tube through the nose to the oropharynx of the child where the tip was retrieved using a small forceps and laryngoscope. The tip was then carefully pulled out through the mouth. The proximal end of the capsule's radioopaque tube was introduced and advanced through the lumen of the Nelaton tube until it came out at the other end. By pulling the proximal extreme of the radioopaque tube the capsule could be placed in the oropharynx, from which it progressed spontaneously overnight. A similar manoeuvre was necessary to retrieve the capsule from the oropharynx.

With the paediatric multipurpose suction biopsy tube, on the other hand, the average time of intubation was between 30 and 45 minutes. Furthermore, two specimens were regularly obtained. The main drawback was the transoral technique followed during intubation. Besides the emotional trauma that it often represented to the child, we soon learned that this approach could not be used in children over $1 \frac{1}{2}$ years of age, as the tube walls were too often damaged by biting, despite the use of heavier sedation and different kinds of oral protectors, including plastic dental plates and short pieces of thick-walled tube covering the biopsy tube.

For all these reasons it was decided to design a tube and capsule which, although retaining some of the characteristics of the multipurpose suction biopsy tube, could be introduced through the nose without trauma and with minimal discomfort to the patient. The final version has proved to be a safe and reliable instrument if carefully used. In the majority of cases the whole procedure was completed while the nasal mucosa was still anaesthetized. The failure rate compares favourably with those reported by other authors with different types of capsules (Anderson, 1966; Brandborg et al, 1959; Crosby and Kugler, 1957).

The use of local anaesthesia in the nasal mucosa made it possible to obtain satisfactory levels of sedation with lower or regular pharmacological doses of the drugs used for sedation. Consequently emotional trauma was decreased. Up to the present, no harmful effects have been observed with the local use of either novesine or xylocaine. We also assume that using metoclopramide helped to shorten the time taken, decreased nausea, and assisted the passage of the tube through the pylorus. Similar experiences have been previously reported by other authors (Mitchell and Parkins, 1969). It is not yet clear why some of the severely malnourished children in this series became progressively resistant to the action of the drugs used for sedation. The fact that this development seemed to be connected with the repeated administration of sedative drugs suggests that it might be due to a drug-induced enhancement of liver microsomal enzymes which will, in turn, alter the susceptibility of children to the action of these drugs (Remmer and Merker, 1963). This possibility still remains to be confirmed through further study.

This study was supported in part by a grant from the Josiah Macy Jr Foundation, New York.

\section{References}

Anderson, C. M. (1966). Intestinal malabsorption in childhood. Arch. Dis. Childh., 41, 571-596.

Brandborg, L. L., Rubin, G. E., and Quinton, W. E. (1959). A multipurpose instrument for suction biopsy of the esophagus, stomach, small bowel and colon. Gastroenterology, 37, 1-16.

Crosby, W. H., and Kugler, H. W. (1957). Intraluminal biopsy of the small intestine: the intestinal biopsy capsule. Amer. $J$. dig. Dis., 2, 236-241.

Israel, R., Hohn, A. R., Black, I. F. S., and Lambert, E. C. (1967). Evaluation of sedation during cardiac catheterization of children. J. Pediat., 70, 407-412.

Mitchell, A. B. S., and Parkins, R. A. (1969). Metoclopramide as an adjunct to small bowel intubation. Gut, 10, 690 .

Remmer, H., and Merker, H. J. (1963). Enzyminduktion und Vermehrung von endoplasmatishem Reticulum in der Leberzelle während der Behandlung mit Phenobarbital (Luminal). Klin.Wschr., 41, 276-283.

Salem, S. N., Salt, R. H., and Truelove, S. C. (1965). Crosby smallintestinal capsule with radio-opaque tube and latex sheath. Gut, 6, 99-100. 